\title{
Development and validation of a novel single nucleotide polymorphism (SNP) panel for genetic analysis of Blastomyces spp. and association analysis
}

Holly M. Frost ${ }^{1,2}$, Jennifer L. Anderson², Lynn Ivacic ${ }^{2}$, Brian L. Sloss ${ }^{3}$, John Embil ${ }^{4}$ and Jennifer K. Meece ${ }^{2^{*}}$

\begin{abstract}
Background: Single nucleotide polymorphism (SNP) genotyping is increasingly being utilized for molecular typing of pathogens and is cost-effective, especially for large numbers of isolates. The goals of this study were 1) to develop and validate a SNP assay panel for genetic analysis of Blastomyces spp., 2) ascertain whether microsatellite genotyping and the SNP genotyping with the developed panel resolve identical genetic groups, and 3) explore the utility of SNPs for examining phylogenetic and virulence questions in humans.

Methods: Three hundred sixty unique Blastomyces spp. isolates previously genotyped with microsatellite markers were genotyped with the MassARRAY ${ }^{\circledR}$ SNP genotyping system (Agena Bioscience ${ }^{\text {TM }}$, San Diego, CA), for a custom panel of 28 SNPs. Clinical presentation data was analyzed for association with SNP variants.

Results: Three hundred twenty-three Blastomyces spp. isolates (90 \%) were successfully genotyped by SNP analysis, with results obtained for at least 27 of 28 assays. For $99.7 \%$ of isolates tested by both genotyping methods, microsatellite genetic group assignment correlated with species assignment based on internal transcribed spacer 2 (ITS2) genotyping, with Group 1 ( $G r$ 1) being equivalent to B. gilchristii and Group 2 ( $G r$ 2) being equivalent to $B$. dermatitidis. Thirteen isolates were genetic hybrids by one or both methods of genotyping and were difficult to assign to a particular genetic group or species. Fifteen SNP loci showed significantly different alleles in cases of pulmonary vs disseminated disease, at a $p$-value of $<0.01$ or less.

Conclusions: This study is the largest genotyping study of Blastomyces spp. isolates and presents a new method for genetic analysis with which to further explore the relationship between the genetic diversity in Blastomyces spp. and clinical disease presentation. We demonstrated that microsatellite Gr 1 is equivalent to B. gilchristii and $\mathrm{Gr} 2$ is equivalent to $B$. dermatitidis. We also discovered potential evidence of infrequent recombination between the two Blastomyces spp. Several Blastomyces spp. SNPs were identified as associated with dissemination or pulmonary disease presentation, but additional work is needed to examine virulence SNPs separately within $B$. dermatitidis and B. gilchristii.
\end{abstract}

Keywords: B. dermatitidis, B. gilchristii, Blastomyces, Genotype, SNP, Microsatellite, Blastomycosis

\footnotetext{
* Correspondence: meece.jennifer@mcrf.mfldclin.edu

${ }^{2}$ Marshfield Clinic Research Foundation, Marshfield Clinic, Marshfield, WI

54449, USA

Full list of author information is available at the end of the article
} 


\section{Background}

Blastomyces spp. are thermally dimorphic fungi endemic to regions of North America with sporadic cases in India, Africa, and South America [1-4]. Genetic typing of Blastomyces spp. isolates using microsatellite markers revealed two distinct genetic groups [5]. Later, significant associations between microsatellite genetic group and clinical disease phenotype were demonstrated in humans, with genetic Gr 1 being associated with isolated pulmonary disease and Gr 2 being associated with cases of disseminated disease [6]. More recently, multilocus sequence typing of Blastomyces spp. isolates has led to the proposal of a cryptic species, $B$. gilchristii, within the group historically referred to as $B$. dermatitidis [7]. In that study, 46 nucleotide polymorphisms were identified within 7 gene regions, with 12 SNPs being determined as diagnostic between $B$. gilchristii and B. dermatitidis. Genotyping of a small number of isolates by both methods indicates that $B$. gilchristii, the newly proposed species, may be equivalent to microsatellite genetic Group 1 with $B$. dermatitidis being equivalent to microsatellite genetic Gr 2 [7, 8]. This suggests that clinical disease variation is potentially associated with species-specific genetic diversity.

SNPs are a valuable tool for studying recombination, rearrangement, relatedness and other genetic processes. In humans, SNPs occur at approximately $1 \mathrm{SNP} /$ kilobase throughout the genome and are responsible for most monogenic disorders [9]. Due to the versatility of SNPs to examine varying genetic questions, they are increasingly being utilized for molecular typing of pathogens [10-13]. SNP genotyping is easily suited to high-throughput testing which is more cost-effective than microsatellite typing and/or Sanger sequencing. In the case of Blastomyces, the ability to determine the species of an isolate from a small number of SNPs is particularly useful given the established associations with clinical features, such as disease dissemination. The goals of this study were 1) to develop and validate a SNP assay panel for genetic analysis of Blastomyces spp. isolates, 2) ascertain whether microsatellite genotyping and SNP genotyping with the developed panel resolve similar phylogenetic groups, and 3) explore the utility of SNPs for examining virulence associations in cases of human disease.

\section{Methods}

\section{Isolates}

Three hundred sixty unique Blastomyces spp. isolates previously extracted [6] and genotyped using 27 polymorphic microsatellite markers [7], were selected for this study. Only isolates with complete microsatellite typing for all 27 markers were included in the study. These included: 295 human isolates, 51 canine isolates, 8 environmental isolates, 4 feline isolates, 1 equine isolate, and 1 alpaca isolate. Twenty isolates were gifted to us from other researchers, three isolates were purchased from ATCC (26199, 18187, and 18188), and the remaining isolates were obtained as part of clinical diagnosis at Diagnostic Services of Manitoba $(n=28)$ or Marshfield Labs ${ }^{\mathrm{TM}}(n=309)$. All isolates were identified as Blastomyces spp. using standard methods, which included culture of the mold form on brain-heart infusion agar with blood at $25{ }^{\circ} \mathrm{C}$ and conversion to the yeast form when incubated in Middlebrook $7 \mathrm{H} 9$ broth at $35{ }^{\circ} \mathrm{C}$. Clinical presentation and mortality data was previously abstracted on 310 of these cases for a former study [6]. Research protocols were approved by the Marshfield Clinic Research Foundation Institutional Review Board. Waiver of informed consent was obtained for retrospective review of clinical information, specimen collection and genotyping.

\section{SNP assay development and genotyping}

Blastomyces spp. isolates were genotyped with the MassARRAY $^{\oplus}$ SNP genotyping system (Agena Bioscience ${ }^{\mathrm{T \mu}}$, San Diego, CA), for a custom panel of 28 single nucleotide polymorphisms (SNPs). To design our custom SNP assay, 21 gene regions were investigated for appropriate polymorphism targets that could be multiplexed into a single-well, high-throughput genotyping platform. The gene regions evaluated included known and potential virulence and housekeeping genes in both coding and non-coding areas. Alignments for each gene target were obtained from publicly available sources, National Center for Biotechnology Information (NCBI) GenBank (available at http://www.ncbi.nlm.nih.gov/genbank/) and the Broad Institute [14], and sequence data generated in our lab (data not shown). One hundred and eight different SNPs and insertion/deletions (INDELs) within 21 gene regions were evaluated for appropriate PCR and extension primer combinations. Allowing the design software to assemble multiple iterations of possible target combinations, a 28plex assay was chosen that included at least one target from each of 19 gene regions (Table 1).

Two-Ten ng of each deoxyribonucleic acid (DNA) sample was amplified in a $5 \mu \mathrm{L}$ reaction containing $1 \mathrm{U}$ of Taq enzyme, $1 \mathrm{X}$ Buffer, $2.0 \mathrm{mM} \mathrm{MgCl}_{2}, 500 \mu \mathrm{M}$ each $\mathrm{dNTP}$ and $0.1 \mu \mathrm{M}$ of each gene-specific forward and reverse primer (Additional file 1). Cycling conditions were $2 \mathrm{~min}$ at $94{ }^{\circ} \mathrm{C}$ followed by 45 cycles of $30 \mathrm{~s}$ at $94{ }^{\circ} \mathrm{C}, 30 \mathrm{~s}$ at $56{ }^{\circ} \mathrm{C}, 60 \mathrm{~s}$ at $72{ }^{\circ} \mathrm{C}$ and a final extension time of 5 min at $72{ }^{\circ} \mathrm{C}$. After PCR amplification, shrimp alkaline phosphatase was added to the samples and incubated for $40 \mathrm{~min}$ at $37{ }^{\circ} \mathrm{C}$. Extension primers, iPLEX enzyme, buffer, and termination mixture of massmodified di-deoxynucleotide triphosphates were added to initiate the iPLEX primer extension reaction. The cycling conditions consisted of a two-step, 200 short 
Table 1 Single nucleotide polymorphisms (SNPs) included in the genotyping panel

\begin{tabular}{|c|c|c|c|c|}
\hline Gene Region & Function & Category & Variant_Base pair location & NCBI reference \\
\hline Chitinase & $\begin{array}{l}\text { Carbohydrate metabolism, } \\
\text { hyphal growth }\end{array}$ & Gene & chit_2396 & XM_002629522.1 \\
\hline Microsatellite $1.32^{\mathrm{a}}$ & N/A & non-gene region & 132GAx11_108 & $N / A^{b}$ \\
\hline BAD1/WI1 & $\begin{array}{l}\text { Surface adhesion, modulate } \\
\text { host inflammatory response }\end{array}$ & Virulence gene ${ }^{c}$ & $\begin{array}{l}\text { BAD1_2556 } \\
\text { BAD1_2850 } \\
\text { BAD1_2869 }\end{array}$ & U37772.1 \\
\hline ADP-ribosylation factor & Vesicular trafficking & Gene & ARF_374 & AY013310.1 \\
\hline ADP-ribosylation factor $65^{\prime}$ UTR & N/A & Untranslated region & arf6_240 & JN561794.1 \\
\hline histidine kinase & $\begin{array}{l}\text { Morphogenesis, cell wall } \\
\text { composition, sporulation }\end{array}$ & Virulence gene $^{d}$ & $\begin{array}{l}\text { drk1_586 } \\
\text { drk1_595 }\end{array}$ & JN561950.1 \\
\hline alpha tubulin & Morphogenesis & Gene & $\begin{array}{l}\text { TUB1_18 } \\
\text { TUB1_277 }\end{array}$ & JN562331.1 \\
\hline orotidine $5^{\prime}$-phosphate decarboxylase & Biosynthesis of pyrimidines & Gene & $\begin{array}{l}\text { pyrF_21 } \\
\text { pyrF_99 }\end{array}$ & JN562191.1 \\
\hline chitin synthase & Cell Wall/exoskeleton scaffolding & Gene & $\begin{array}{l}\text { chs2_203 } \\
\text { chs2_290 }\end{array}$ & JN561872.1 \\
\hline fatty acid desaturase & Membrane fluidity, thermotolerance & Gene & fads_622 & JN562028.1 \\
\hline $\begin{array}{l}\text { internal } \\
\text { transcribed spacer } 2 \text { of rDNA }\end{array}$ & N/A & Gene & ITS2_19 & JN562151.1 \\
\hline urease & $\begin{array}{l}\text { Ammonia production, protection } \\
\text { from phagocytes }\end{array}$ & Gene & urease_1503 & XM_002623809.1 \\
\hline alpha-[1, 3]-glucan synthase & $\begin{array}{l}\text { Cell wall biogenesis, block } \\
\text { host recognition }\end{array}$ & Gene & $\begin{array}{l}\text { alpha1_3glucan_2360 } \\
\text { alpha1_3glucan_2386 }\end{array}$ & XM_002629303.1 \\
\hline beta-glucosidase & Breaks down cellulose & Gene & $\begin{array}{l}\text { b-glucosidase_966 } \\
\text { b-glucosidase_1243 }\end{array}$ & XM_002621346.1 \\
\hline septin-1 & $\begin{array}{l}\text { Filament formation, scaffold, } \\
\text { sporulation }\end{array}$ & Gene & septin1_1251 & XM_002628186.1 \\
\hline heat shock protein & Thermotolerance & Gene & hsp_764 & XM_002624824.1 \\
\hline DNA Lyase & DNA repair, Flanks mating locus & Gene & APN2_1016 & XM_002623165.1 \\
\hline Acetate- coA ligase & Metabolism & Gene & CoAligase_346 & XM_002626273.1 \\
\hline tryptophan tRNA ligase & ATP binding & Gene & trypt-lig_922 & XM_002620210.1 \\
\hline tyrosinase & Involved in melanin synthesis & Gene & tyrosinase_759 & XM_002623880.1 \\
\hline
\end{tabular}

${ }^{a}$ Microsatellite locus 5, Meece et al. Applied and Environmental Microbiology 17:5123-5131

${ }^{\mathrm{b}}$ Sequence not publically available

'Brandhorst et al. J Biol Chem 275:7925-7934 and Wüthrich et al. Med Mycol 44:41-49

${ }^{\mathrm{d}}$ Nemecek et al. Science 312:583-588

eDiagnostic SNPs according to Brown et al. PLoS One 8:e59237

cycle program with one loop of 5 cycles inside a loop of 40 cycles. The sample was denatured at $94{ }^{\circ} \mathrm{C}$ for $30 \mathrm{~s}$, followed by 5 cycles of annealing at $52{ }^{\circ} \mathrm{C}$ for $5 \mathrm{~s}$ and extended at $80{ }^{\circ} \mathrm{C}$ for $5 \mathrm{~s}$. The five annealing and extension cycles with the single denaturing step was repeated 40 times, for a total number of 200 annealing and extension cycles. MassEXTEND clean resin was added to each reaction to remove extraneous salts that interfere with matrix assisted laser desorption ionization time-of-flight (MALDI-TOF) analysis. Fifteen $\mathrm{nL}$ of the sample was transferred from the plate and spotted onto a matrix pad of the SpectroCHIP array. Genotypes were determined by mass correlations on the MALDI-TOF mass spectrometer.

\section{SNP panel validation}

Twelve isolates ( 9 human isolates, 1 environmental isolate, 1 canine isolate, and 1 alpaca isolate) with previously obtained sequence data (available at NCBI, Table 1, and generated in our lab, data not shown), demonstrating known allelic diversity, were chosen for assay validation, to include examples of expected alleles at a subset of the targets. These 12 validation samples had been sequenced in-house for previous studies and 102 SNP alleles were known on these samples at the 28 loci included in the design. Comparison of previous in-house sequencing results with our iPLEX SNP genotyping was used to validate the accuracy of calls. Each validation sample was assayed in multiple batches of testing to confirm the precision and 
reproducibility of the genotyping results. Validation samples with missing alleles and inconsistent results were reextracted to investigate sample quality and amplification inhibition. In addition to up-front assay validation, 11 duplicate samples were embedded in the final genotyping run, blinded to the genotyping technician.

\section{SNP panel analysis}

Blastomyces spp. isolates with more than 1 missing allele or low probability call, as defined by the instrument software, (4\% missing genotype) were excluded from the SNP portion of the study, in order to avoid classification bias [15]. Species assignment of each isolate was based on SNP ITS2_19 [7]. Allele frequencies, expressed as percentages, were calculated for each SNP separately between the Blastomyces spp.

\section{Comparison of microsatellite and SNP genotyping}

For both microsatellite typing and SNP typing data, haplotypes were ascertained by identifying matching samples, and subsumed to a single representative using Genetic Analysis in Excel v6.41 [16]. Genetic structure among the samples was analyzed separately for each genotyping method using principle coordinate analysis (PCoA) of the standardized covariance of the haplotypic genetic distance as performed in Genetic Analysis in Excel v6.41. All individual genotyping markers were weighted equally for analysis of both microsatellite typing and SNP typing data. The first and second principle coordinate were plotted to graphically illustrate clusters of haplotypes. For microsatellite data, the Bayesian approach of the program STRUCTURE [17] was used to predict the minimum number of genetic units or clusters within the composite data. Analysis settings included $K$ (the putative number of genetic groups) ranging from 1 to 12 , the use of the admixture model, correlated allele frequencies between populations, lambda of one, and the degree of admixture (alpha) was inferred from the data as advised by the software's manual. The burn-in was set at 100,000 repetitions and the length of each iteration was 100,000 repetitions with five iterations of each $K$. The method of Evanno et al. [18] as estimated using Structure Harvester [19] was used to assess the most likely $K$ given the data in conjunction with the mean and variance of the $\ln$ probability of $K$. STRUCTURE output was used to assign individual haplotypes to microsatellite genetic group. Locus-specific diversity measures of the microsatellite genetic groups included: number of alleles, number of unique alleles, and the size and frequency of the most common allele. Genetic group assignment by microsatellite analysis and species assignment using the previously described diagnostic SNP included in the panel [7] were compared for correlation.

\section{SNP associations in human isolates}

For human isolates with both SNP genotyping and clinical data available, associations between SNP and disease presentation were analyzed using a Pearson's chi-square test, with $\alpha=0.05$.

\section{Results \\ SNP panel validation}

We observed $100 \%$ concordance between Sanger sequencing and SNP calls on the 102 previously known alleles on the 12 isolate validation panel. Validation test results were $100 \%$ reproducible when repeated in multiple batches, demonstrated good amplification, and revealed clear-cut genotyping results with all 28 assays. Furthermore, the 11 duplicate samples embedded in the genotyping project showed $100 \%$ agreement for all SNP calls.

\section{SNP panel analysis}

Three hundred twenty-three Blastomyces spp. isolates (90 \%) were successfully genotyped by SNP analysis, with results obtained for at least 27 of 28 assays. The remaining 37 isolates were excluded from the SNP portion of the study due to no amplification $(n=7)$, multiple no call results $(n=20)$, or multiple heterozygous calls $(n=10)$. Results of SNP genotyping are shown in Table 2. The tyrosinase_759 SNP was unable to be genotyped on 9 isolates, making it the least robust assay. Three potential SNPs (chs2_290, fads_622, TUB1_277), designed from GenBank sequences, showed no sequence variation in the isolates tested and were excluded from further analysis. The remaining SNPs were bi-allelic as expected based on design sequences, with the exception of arf6_240, which we discovered to be tri-allelic. Three polymorphisms (ITS2_19, drk1_586, and drk1_595) described by Brown et al. [7] as being diagnostic between $B$. dermatitidis and B. gilchristii were included in the assay. We found that results from genotyping of drk1_586 and drk1_595 SNPs were not in agreement with ITS2_19, as far as species assignment, on 2 isolates in this study (BD9911 and BD0503). Alternatively, results from SNPs trypt-lig_922 and CoAligase_346 assigned all 323 isolates into B. gilchristii and B. dermatitidis consistent with ITS2_19 genotyping. Based on ITS2_19, 146 (45\%) of the isolates in this study were B. dermatitidis with the remaining 177 isolates (55\%) being B. gilchristii. B. gilchristii isolates showed low allelic diversity (frequency of $\geq 98 \%$ for a single allele) at all but 1 locus, chs2_203, which demonstrated a unique allele $(\mathrm{G})$ at a frequency of $21 \%$. The majority of the diversity at this locus $(73 \%)$ was due to 27 Canadian B. gilchristii isolates, which all had the $\mathrm{G}$ allele. Alternatively, $B$. dermatitidis isolates showed much more allelic diversity, with 7 loci exhibiting frequencies of $\leq 90 \%$ for a single allele. At most SNP loci (15 of 25), the most frequent allele differed between $B$. dermatitidis and B. gilchristii. 
Table 2 SNP genotyping results for 323 isolates, by Blastomyces spp. species (ITS2_19)

\begin{tabular}{|c|c|c|c|c|c|}
\hline \multirow[t]{2}{*}{ Marker } & \multirow[t]{2}{*}{ Allele } & B. gilchristii & (\%) & B. dermatitidis & $(\%)$ \\
\hline & & \multicolumn{2}{|l|}{ ITS2_19 C $(n=177)$} & \multicolumn{2}{|l|}{ ITS2_19 Ta $(n=146)$} \\
\hline \multirow[t]{2}{*}{ 132GAx11_108 } & A & 176 & (99) & 14 & {$[10]$} \\
\hline & G & 1 & [1] & 132 & $(90)$ \\
\hline \multirow[t]{2}{*}{ alpha1_3glucan_2360 b } & $A$ & $1^{c}$ & [1] & 62 & $(42)$ \\
\hline & C & 176 & (99) & 84 & $(58)$ \\
\hline \multirow[t]{2}{*}{ alpha1_3glucan_2386 b } & C & $1^{c}$ & [1] & 146 & $(100)$ \\
\hline & G & 176 & (99) & 0 & $(0)$ \\
\hline \multirow[t]{2}{*}{ APN2_1016 } & C & 176 & (99) & 134 & (92) \\
\hline & $\mathrm{T}$ & $1^{c}$ & [1] & 12 & {$[8]$} \\
\hline \multirow[t]{2}{*}{ ARF_374 } & G & 176 & (99) & 1 & {$[1]$} \\
\hline & A & 0 & (0) & 145 & (99) \\
\hline \multirow[t]{3}{*}{ arf6_240 } & A & 176 & (99) & 4 & {$[3]$} \\
\hline & G & 1 & [1] & 133 & $(91)$ \\
\hline & C & 0 & (0) & 9 & {$[6]$} \\
\hline \multirow[t]{2}{*}{ BAD1_4 } & $A$ & 176 & (99) & $1^{d}$ & {$[1]$} \\
\hline & G & $1^{c}$ & [1] & 145 & (99) \\
\hline \multirow[t]{2}{*}{ BAD1_8 ${ }^{b}$} & $A$ & $1^{c}$ & [1] & 145 & (99) \\
\hline & $T$ & 176 & (99) & $1^{d}$ & {$[1]$} \\
\hline \multirow[t]{2}{*}{ BAD1_9 $9^{b}$} & C & 176 & (99) & $1^{d}$ & {$[1]$} \\
\hline & G & $1^{c}$ & [1] & 145 & (99) \\
\hline \multirow[t]{2}{*}{ b-glucosidase_1243b } & G & 177 & (100) & 96 & $(66)$ \\
\hline & $A$ & 0 & (0) & 50 & (34) \\
\hline \multirow[t]{3}{*}{ b-glucosidase_966 } & A & 175 & (99) & 0 & $(0)$ \\
\hline & G & $1^{c}$ & [1] & 145 & (99) \\
\hline & N/A & 1 & [1] & $1^{d}$ & {$[1]$} \\
\hline \multirow[t]{2}{*}{ chit_2396b } & C & 177 & (100) & 109 & $(75)$ \\
\hline & A & 0 & (0) & 37 & $(25)$ \\
\hline \multirow[t]{2}{*}{ chs2_203 } & $C$ & 140 & (79) & 146 & $(100)$ \\
\hline & G & $37^{e}$ & [21] & 0 & (0) \\
\hline \multirow[t]{2}{*}{ chs2_290 } & C & 177 & (100) & 146 & $(100)$ \\
\hline & G & 0 & (0) & 0 & $(0)$ \\
\hline \multirow[t]{2}{*}{ CoAligase_346 } & G & 177 & (100) & 0 & $(0)$ \\
\hline & A & 0 & (0) & 146 & $(100)$ \\
\hline \multirow[t]{2}{*}{ drk1_586 } & C & 176 & (99) & 1 & {$[1]$} \\
\hline & $\mathrm{T}$ & 1 & [1] & 145 & (99) \\
\hline \multirow[t]{2}{*}{ drk1_595 a } & A & 176 & (99) & $1^{d}$ & {$[1]$} \\
\hline & G & $1^{c}$ & [1] & 145 & (99) \\
\hline \multirow[t]{2}{*}{ fads_622 ${ }^{f}$} & $T$ & 177 & (100) & 146 & (100) \\
\hline & $C$ & 0 & (0) & 0 & (0) \\
\hline \multirow[t]{2}{*}{ hsp_764 } & A & 176 & (99) & 13 & [9] \\
\hline & $C$ & $1^{c}$ & [1] & 133 & (91) \\
\hline \multirow[t]{2}{*}{ pyrF_21 } & A & 177 & (100) & 140 & (96) \\
\hline & $C$ & 0 & (0) & 6 & {$[4]$} \\
\hline pyrF_99 & $C$ & 177 & (100) & 144 & (99) \\
\hline
\end{tabular}


Table 2 SNP genotyping results for 323 isolates, by Blastomyces spp. species (ITS2_19) (Continued)

\begin{tabular}{|c|c|c|c|c|c|}
\hline & G & 0 & (0) & 2 & {$[1]$} \\
\hline \multirow[t]{2}{*}{ septin1_1251 } & A & 175 & (99) & 17 & {$[12]$} \\
\hline & G & 2 & {$[1]$} & 129 & (88) \\
\hline \multirow[t]{2}{*}{ trypt-lig_922 } & C & 177 & $(100)$ & 0 & (0) \\
\hline & G & 0 & (0) & 146 & (100) \\
\hline \multirow[t]{2}{*}{ TUB1_18 } & C & 177 & $(100)$ & 144 & (99) \\
\hline & $\mathrm{T}$ & 0 & (0) & 2 & [1] \\
\hline \multirow[t]{2}{*}{ TUB1_277 ${ }^{f}$} & C & 177 & $(100)$ & 146 & (100) \\
\hline & G & 0 & (0) & 0 & (0) \\
\hline \multirow[t]{3}{*}{ tyrosinase_759 } & $\mathrm{T}$ & 174 & (98) & 132 & $(90)$ \\
\hline & A & 0 & (0) & 8 & {$[5]$} \\
\hline & N/A & 3 & [2] & 6 & {$[4]$} \\
\hline \multirow[t]{2}{*}{ urease_1503 } & A & 177 & $(100)$ & 69 & $(47)$ \\
\hline & G & 0 & (0) & 77 & (53) \\
\hline
\end{tabular}

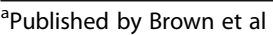

${ }^{b} S N P$ results in amino acid change

Isolate BD9911

dsolate BD0503

eIncludes 27 Canadian isolates

${ }^{f}$ Expected SNP based on genbank sequence

\section{Comparison of microsatellite and SNP genotyping}

The 323 Blastomyces spp. isolates with successful SNP genotyping were subsumed to 73 unique haplotypes (Additional file 2). SNP PCoA, shown in Fig. 1a demonstrates clustering of the haplotypes into 2 primary groups, with the exception of 2 intermediate haplotypes, isolates BD9911 and BD0503 described above. The first primary axis (Coordinate 1 ) explained $69 \%$ of the variance between $B$. dermatitidis and B. gilchristii.

Microsatellite typing data was available on all 360 isolates, including the 37 isolates that were excluded from the SNP portion of the study. Two hundred twenty-four unique microsatellite haplotypes were identified. For microsatellite PCoA, the first primary axis (Coordinate 1) explained $25 \%$ of the variance between the genetic groups (Fig. 1b). STRUCTURE analysis of the unique microsatellite haplotypes supported two genetic units in the data $(K=2)$ based on the method of Evanno et al. [18] and the linearity and variance of $\ln P(D)$. The individual ancestry of each haplotype based on $K=2$ revealed 62 haplotypes representing Gr 1 isolates and 162 haplotypes representing Gr 2 isolates. Examination of microsatellite group-specific allelic diversity revealed significant difference between the two genetic groups (Table 3). Gr 1 isolates show low allelic diversity, ranging from 1-7 alleles/locus (avg 3.7). Gr 2 isolates exhibit more polymorphism, ranging from 4-18 alleles/locus (avg 10.6). Across all loci, Gr 1 contained 14 alleles not present in Gr 2; Gr 2 contained 200 alleles, not represented in Gr 1. Comparison of microsatellite and SNP genotyping showed that all isolates assigned to microsatellite $\mathrm{Gr} 1$ by
STRUCTURE were B. gilchristii by ITS2_19 genotyping and all isolates assigned to microsatellite Gr 2 by STRUCTURE were B. dermatitidis, with the exception of BD9911. Within the data were 13 isolates with a majority q-value (genetic membership threshold) of $\leq 90 \%$ (Fig. 2), by STRUCTURE analysis of microsatellite genotyping. Two intermediate isolates had nearly equal genetic membership in both groups; identified as BD9911 (genetic membership, $55 \%$ Gr 2 and $45 \%$ Gr 1) and BD0403 (genetic membership, $52 \%$ Gr 1 and $48 \%$ Gr 2).

\section{SNP associations in human isolates}

SNP genotyping and clinical abstraction data were available on 240 human isolates. This was comprised of 151 B. gilchristii isolates (14 cases of disseminated disease and 137 cases of exclusively pulmonary disease) and 89 cases of $B$. dermatitidis (31 cases of disseminated disease and 58 cases of exclusively pulmonary disease). When analyzed globally without regard to species, SNP alleles were significantly different in cases of pulmonary vs disseminated disease, at a $p$-value of $<0.01$ or less, for the following SNPs: 132GAx11_108, alpha1_3glucan_2360, alpha1_3glucan_2386, ARF_374, BAD1_4, BAD1_8, BAD1_9, b-glucosidase_966, CoAligase_346, drk1_586, drk1_595, hsp764, ITS2_19, septin1_1251, and tryptlig_922 (Additional file 3).

\section{SNPs of interest in B. dermatitidis and B. gilchristii}

Seven SNP loci in B. dermatitidis demonstrated within species allelic diversity (frequency of $\leq 90 \%$ for a single allele). Only 6 of these were located within a gene 


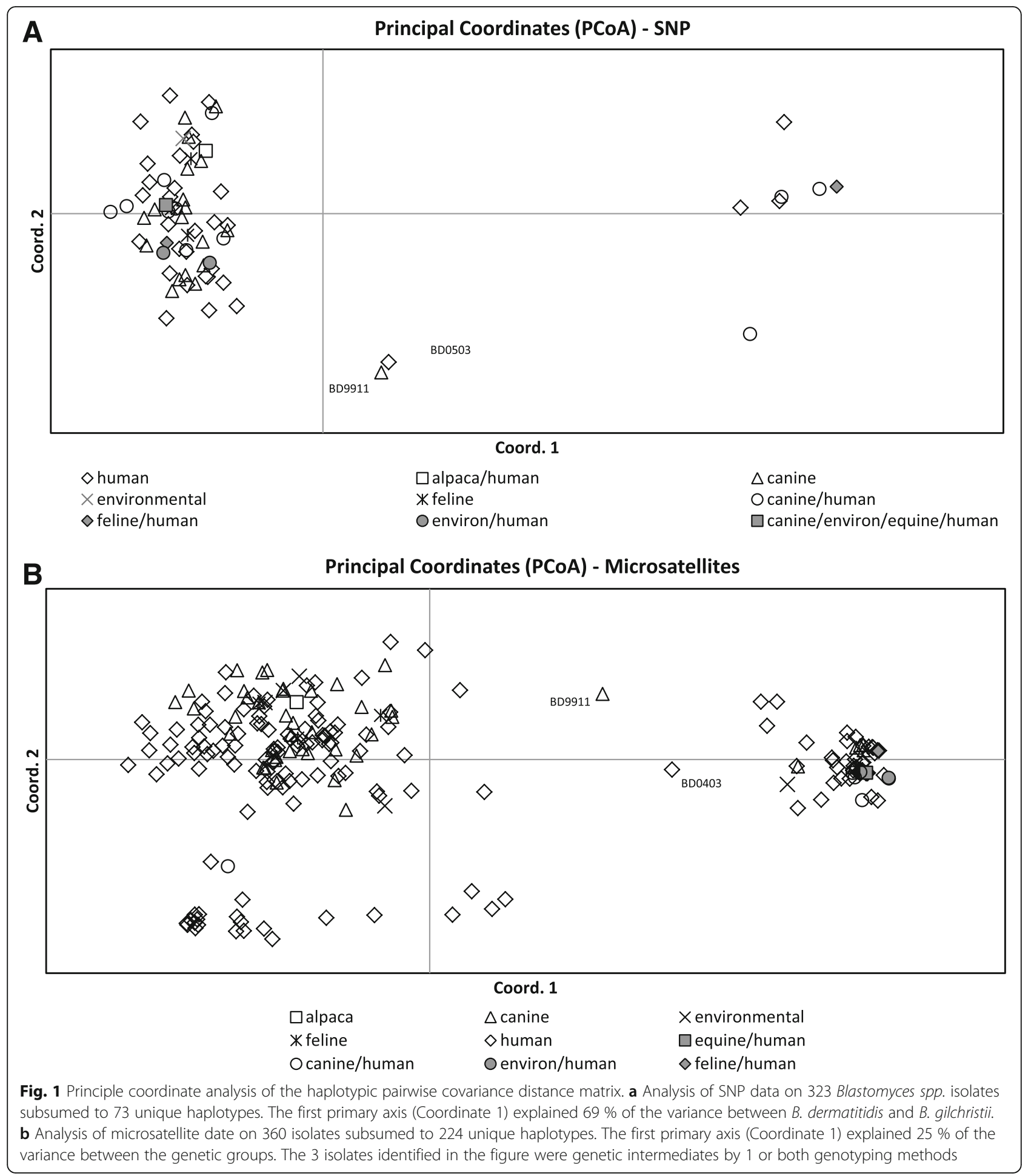

coding region. One SNP locus in B. gilchristii met the above criteria for diversity and was also in a coding region. These SNPs were not analyzed for association with disseminated and pulmonary disease presentation within each Blastomyces spp. separately due to limited statistical power. We did observe that the A urease_1503 allele (frequency $65 \%$ ) and G septin1_1251 allele (frequency $90 \%$ ) were more often observed in B. dermatitidis isolates resulting in cases of disseminated disease, though these SNPs do not represent amino acid changes. In addition, the A alpha1_2glucan_2360 allele, which does result in an amino acid change, showed a slightly 
Table 3 Summary of alleles by microsatellite genetic group

\begin{tabular}{|c|c|c|c|c|c|c|c|c|}
\hline \multicolumn{5}{|c|}{ Group 1} & \multicolumn{4}{|c|}{ Group 2} \\
\hline Locus & $\mathrm{n}_{\mathrm{A}}^{\mathrm{a}}$ & Unique & Most Common Allele ${ }^{b}$ & Freq $^{c}$ & $\overline{n_{A}^{a}}$ & Unique & Most Common Allele ${ }^{b}$ & Freq $^{c}$ \\
\hline 1 & 3 & 0 & 250 & 0.73 & 4 & 1 & 250 & 0.66 \\
\hline 2 & 4 & 0 & 213 & 0.96 & 9 & 5 & 203 & 0.34 \\
\hline 3 & 5 & 0 & 199 & 0.93 & 17 & 12 & 209 & 0.29 \\
\hline 4 & 6 & 0 & 219 & 0.77 & 10 & 4 & 229 & 0.40 \\
\hline 5 & 3 & 0 & 214 & 0.98 & 10 & 7 & 214 & 0.42 \\
\hline 6 & 7 & 3 & 279 & 0.47 & 9 & 5 & 265 & 0.58 \\
\hline 7 & 4 & 2 & 273 & 0.56 & 8 & 6 & 255 & 0.66 \\
\hline 8 & 4 & 0 & 214 & 0.89 & 12 & 8 & 208 & 0.49 \\
\hline 9 & 4 & 1 & 194 & 0.93 & 9 & 6 & 194 & 0.53 \\
\hline 10 & 3 & 0 & 206 & 0.96 & 10 & 7 & 206 & 0.55 \\
\hline 11 & 4 & 0 & 155 & 0.98 & 16 & 12 & 173 & 0.16 \\
\hline 12 & 4 & 0 & 251 & 0.79 & 10 & 6 & 249 & 0.23 \\
\hline 13 & 4 & 1 & 192 & 0.98 & 8 & 5 & 184 & 0.36 \\
\hline 14 & 3 & 0 & 263 & 0.99 & 9 & 6 & 269 & 0.38 \\
\hline 15 & 3 & 0 & 198 & 0.99 & 8 & 5 & 202 & 0.32 \\
\hline 16 & 5 & 0 & 254 & 0.82 & 15 & 10 & 258 & 0.49 \\
\hline 17 & 3 & 0 & 232 & 0.98 & 9 & 6 & 234 & 0.34 \\
\hline 18 & 3 & 0 & 200 & 0.95 & 18 & 15 & 224 & 0.15 \\
\hline 19 & 4 & 3 & 231 & 0.94 & 9 & 8 & 241 & 0.26 \\
\hline 20 & 4 & 2 & 221 & 0.96 & 13 & 11 & 235 & 0.34 \\
\hline 21 & 3 & 0 & 189 & 0.92 & 12 & 9 & 197 & 0.27 \\
\hline 22 & 1 & 0 & 200 & 1.00 & 13 & 12 & 206 & 0.23 \\
\hline 23 & 4 & 0 & 220 & 0.98 & 7 & 3 & 222 & 0.45 \\
\hline 24 & 3 & 0 & 165 & 0.98 & 8 & 5 & 167 & 0.53 \\
\hline 25 & 3 & 0 & 250 & 0.98 & 8 & 5 & 256 & 0.35 \\
\hline 26 & 4 & 2 & 179 & 0.78 & 14 & 12 & 167 & 0.44 \\
\hline 27 & 3 & 0 & 235 & 0.59 & 12 & 9 & 233 & 0.34 \\
\hline
\end{tabular}

${ }^{a} n_{A}=$ number of alleles

$b_{\text {amplicon size in base pairs }}$

${ }^{c}$ Freq $=$ frequency of the most common allele

higher frequency (52\%) in cases of disseminated disease caused by $B$. dermatitidis. In B. gilchristii, the $\mathrm{G}$ allele at locus chs2_203 was found at a higher frequency in patients with disseminated disease (43\%) as compared to pulmonary disease $(18 \%)$ and in cases resulting in death (38\%) as compared to no death (19\%). All 27 Canadian B. gilchristii isolates in this study exhibited the $\mathrm{G}$ allele at this locus.

\section{Discussion}

This manuscript describes the development of a SNP panel for genotyping Blastomyces spp. isolates. SNP genotyping revealed more allelic diversity in $B$. dermatitidis isolates than $B$. gilchristii isolates, which is consistent with previous studies [7]. In $B$. dermatitidis isolates, 7 markers demonstrated a frequency of $\leq 90 \%$ for a given allele in the population. B. gilchristii demonstrates this level of diversity in only 1 marker (chs2_203), with the remaining 27 markers showing $>98 \%$ of the population having a single allele for a given marker. A large percentage of the diversity shown in B. gilchristii at SNP chs2_203 is due to 27 Canadian isolates (100\% of the Canadian B. gilchristii isolates) that have the G allele at that locus. Only 10 additional Wisconsin B. gilchristii isolates ( 3 canine and 7 human) had the G allele at locus chs2_203 suggesting regional genetic differences. Interestingly, marker chs2_203, which demonstrates allelic diversity in B. gilchristii, seems to be fixed in B. dermatitidis.

SNP genotyping with this panel largely supports the division of Blastomyces spp. into 2 genetic groups, with most isolates having alleles that are characteristic of their species group. For 322/323 (99.7 \%) isolates tested 


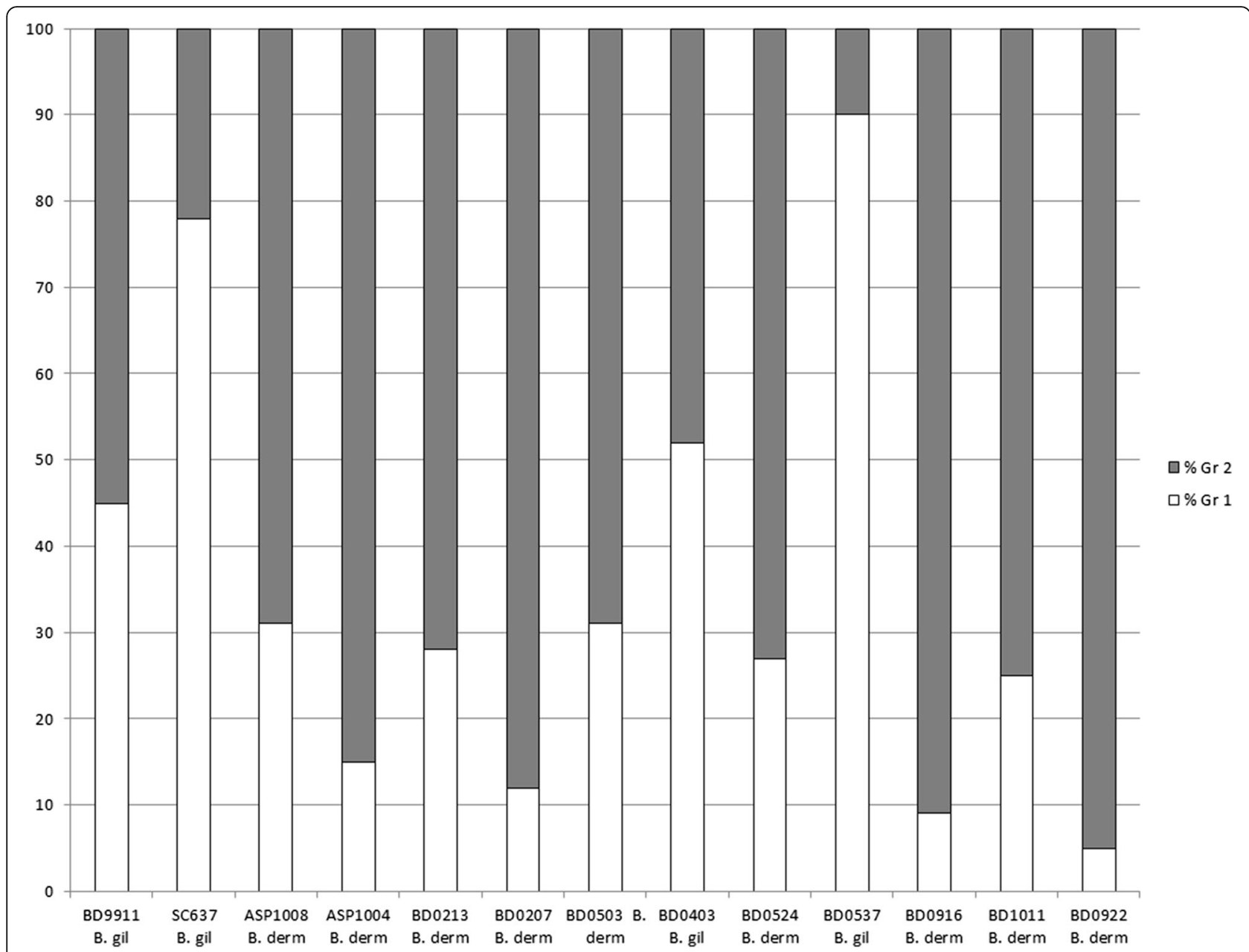

Fig. 2 Mean q-values (genetic membership threshold) from microsatellite STRUCTURE analysis for 13 "hybrid" haplotypes with $\mathrm{K}=2$

by both genotyping methods in this study, microsatellite genetic group assignment correlated with species assignment based on ITS2_19 genotyping, with Gr 1 being equivalent to $B$. gilchristii and Gr 2 being equivalent to $B$. dermatitidis. Several isolates in this study were particularly interesting as they appear to be genetic hybrids as determined by one or both methods of genotyping (Fig. 2). It is clear that these isolates do not fit neatly into the previously defined microsatellite or species groups and that both of these genotyping methods may be detecting hybridization between $B$. dermatitidis and B. gilchristii. Brown et al. [7] were only able to detect genetic recombination when it was assessed separately within each Blastomyces $s p$. This may be because only 78 samples were analyzed in that study. In this study of 323 isolates, we found evidence of potential recombination between the two species in a small percentage of isolates. In order to accurately assess genetic recombination between the two species, a larger study of more genetic intermediate isolates would be necessary.
Our SNP assay did not resolve the same level of genetic variability within Blastomyces spp. isolates as compared to our microsatellite assay. This is most certainly due to the higher mutation rate of microsatellite regions. In fact, previous studies for forensics applications in humans have shown that 3-4 SNPs are comparable to the genetic information in 1 microsatellite marker [20, 21]. Taking this into account, our assays were not directly comparable since they both had about the same number of loci. Furthermore the target regions of the genome were quite different between the assays with almost all of the targets included on the SNP panel being in coding regions. We must also point out that our SNP assay is multiplexed in a single low-volume reaction, whereas microsatellite typing in our lab is performed in single individual reactions for each locus. A SNP genotyping success rate of $90 \%$ on isolates in this study can be partially explained by the fact that multiplex assays are more sensitive to factors such as degraded DNA and carryover of inhibitors, both of which we observed to a small degree in our samples. In 
summary, microsatellite typing had the disadvantages of being labor intensive, low throughput, and expensive, yet provides the most sensitivity for examining population genetics questions. In contrast, SNP genotyping was more cost effective, high throughput and could be used to target gene coding regions, but was less sensitive for resolving genetic differences that impact population structure. However, both methods largely discriminate the vast majority of isolates into one of the two distinct Blastomyces spp.

A small number of isolates, dropped from the analysis portion of the study, produced heterozygous SNP genotyping calls. In $\sim 10 \%$ of the DNA samples tested in this study, we observed a 260/280 ratio of $<1.8$ (indicating protein or phenol carry-over) or $>2.0$ (indicating RNA carry-over), evidence of inhibitors, and/or degraded DNA. This may be an explanation for the isolates with 1-2 "aggressive or low-probability" heterozygous calls as defined by the software. Three of the isolates had heterozygous calls for almost half of the loci, including the ITS2_19. For those isolates we propose that the patient had a dual infection, which has been previously documented in the literature by us and another group [5, 22]. It is possible that we have more isolates in our biobank that represent dual infections as we have no way of knowing how often this occurs in patients.

The discovery of heterozygous SNP calls for some of our isolates made us re-examine our hybrid isolates. None of our hybrid isolates had any heterozygous SNP calls and none of the isolates with heterozygous SNP calls were hybrids by STRUCTURE analysis. Furthermore, we verified the mating type of each of the hybrid isolates, tested previously for another study [5]. All thirteen hybrid isolates (Fig. 2) were previously tested by PCR for mating type and all were positive for only 1 allele, either the high mobility group (HMG) locus or alpha-box locus.

SNP analysis of all Blastomyces spp. isolates in human cases revealed significant association between SNP and disease location (exclusive pulmonary or disseminated) in 15 of the 28 loci (Additional file 3). We expected this result due to previously published associations between the divergent genetic groups of Blastomyces spp. and clinical features [6]. These results are included as supplementary since they represent replicated support of the already established association between genetics and virulence. In fact, among the SNPs which are significantly different between pulmonary and disseminated disease are, a SNP within a microsatellite marker previously used for the association study referenced above [6] and the ITS2 SNP reported by Brown et al. [7] as diagnostic between $B$. dermatitidis and B. gilchristii.

SNP association analysis in each of the Blastomyces spp. separately, was unable to be performed due to limited statistical power, although several SNPs of interest were identified in B. dermatitidis and B. gilchristii for future studies. The A urease_1503, G septin1_1251, and A alpha1_2glucan_2360 alleles were more frequent in cases of disseminated disease caused by $B$. dermatitidis. In B. gilchristii, the G allele at locus chs2_203 was found at a higher frequency in patients with disseminated disease as compared to pulmonary disease and in cases resulting in death as compared to no death. Notably death and dissemination in B. gilchristii infections did not occur together frequently (only 2 of 13 cases) therefore these cases are not synonymous. It is important to point out the impact of the Canadian isolates of $B$. gilchristii on SNP allele frequency at locus chs2_203. All 27 Canadian B. gilchristii isolates in this study exhibited the $\mathrm{G}$ allele at this locus, which is represented in the United States (US) B. gilchristii isolates at a much smaller frequency, about $3 \%$. Only 17 Canadian $B$. gilchristii isolates are represented in Additional file 3, due to incomplete clinical data on some isolates. The clinical data that was available on these isolates showed a higher incidence of both disseminated disease $(n=5$, $30 \%)$ and death $(n=4,24 \%)$ than US isolated $B$. gilchristii cases in this study, or previously published studies [6]. This SNP association is probably not very meaningful from a virulence standpoint as it appears in a housekeeping gene and does not result in an amino acid change.

This study was limited to Blastomyces spp. isolates with previous microsatellite typing and only represents a limited geographic range of the organism. Additionally, SNP association analysis to clinical presentation was unable to be evaluated in B. dermatitidis and B. gilchristii separately. Despite this, the results of this study provide another tool for examining the genetic diversity of Blastomyces spp.

\section{Conclusions}

This is the largest genotyping study of Blastomyces spp. isolates and presents a new method for genetic analysis with which to further explore the relationship between the genetic diversity in Blastomyces spp. and clinical disease presentation. We demonstrated that for $99.7 \%$ of isolates tested by both genotyping methods in this study, microsatellite genetic group assignment correlated with species assignment based on ITS2_19 genotyping, with Gr 1 being equivalent to B. gilchristii and Gr 2 being equivalent to $B$. dermatitidis. We also discovered potential evidence of infrequent recombination between the 2 Blastomyces spp. In addition, several Blastomyces spp. SNPs were identified as associated with dissemination or pulmonary disease presentation, but additional work is needed to examine virulence SNPs separately within B. dermatitidis and B. gilchristii. 


\section{Additional files}

Additional file 1: Primers for the primary PCR amplification of each target in the 28 assay panel. List of forward and reverse primers for amplification of each SNP target. (DOCX $15 \mathrm{~kb}$ )

Additional file 2: SNP genotyping haplotypes. Raw SNP genotyping data obtained from all isolates, subsumed to 73 unique haplotypes. (XLSX $21 \mathrm{~kb})$

Additional file 3: SNP associations for disease presentation and mortality for 240 human isolates, globally for Blastomyces spp. Statistical analysis of SNP status by pulmonary or disseminated disease presentation. (DOCX $19 \mathrm{~kb}$ )

\section{Abbreviations}

DNA: Deoxyribonucleic acid; Gr 1: Group 1; Gr 2: Group 2; HMG: High mobility group; ITS2: Internal transcribed spacer 2; NCBI: National center for biotechnology information; PCOA: Principle coordinate analysis; SNP: Single nucleotide polymorphism; US: United States

\section{Acknowledgements}

We would like to thank Steven Schrodi, PhD for advice on statistical analysis.

\section{Funding}

This work was supported by the Marshfield Clinic Research Foundation [FRO30114 to HMF].

\section{Availability of data and material}

All data generated or analysed during this study are included in this published article [and its Additional files]

\section{Authors' contributions}

HMF and JKM were responsible for the study concept and its design. $J L A, L I, J E$ and JKM participated in the acquisition of data. Statistical analysis and interpretation of data were done by HMF, JLA, BS, and JKM. HMF, JLA, and JKM drafted the manuscript. All authors read and approved of the final manuscript.

\section{Competing interests}

The authors declare that they have no competing interests.

\section{Consent for publication}

All authors have read and agreed to Biomed requirements on publications ethics, and conduct, reporting, editing, and publications recommendations. The manuscript conforms to the journal's policies.

\section{Ethics approval and consent to participate}

The study was approved by the Marshfield Clinic Research Foundation Institutional Review Board. This study was exempted from the request of the patient consent.

\section{Author details}

'Department of Pediatrics, Marshfield Clinic, Minocqua, WI 54548, USA. ${ }^{2}$ Marshfield Clinic Research Foundation, Marshfield Clinic, Marshfield, W 54449, USA. ${ }^{3}$ College of Natural Resources, University of Wisconsin-Stevens Point, Stevens Point, WI 54481, USA. ${ }^{4}$ Health Sciences Centre, University of Manitoba, Winnipeg, Manitoba, Canada.

Received: 4 March 2016 Accepted: 17 September 2016

Published online: 23 September 2016

\section{References}

1. Bradsher RW, Chapman SW, Pappas PG. Blastomycosis. Infect Dis Clin North Am. 2003;17:21-40. vii.

2. Kwon-Chung K, Bennett J. Medical mycology. 2nd ed. Philadelphia: Lea \& Febiger; 1992. p. 248-79.

3. Mandell G, Bennett J, Dolin R. Principles and practice of infectious diseases. 6th ed. New York: Churchill Livingston; 2000. p. 2733-46.
4. Carman WF, Frean JA, Crewe-Brown HH, Culligan GA, Young CN. Blastomycosis in Africa. A review of known cases diagnosed between 1951 and 1987. Mycopathologia. 1989;107:25-32.

5. Meece JK, Anderson JL, Fisher MC, Henk DA, Sloss BL, et al. Population genetic structure of clinical and environmental isolates of Blastomyces dermatitidis based on 27 polymorphic microsatellite markers. Appl Environ Microbiol. 2011;17:5123-31.

6. Meece JK, Anderson JL, Gruszka S, Sloss BL, Sullivan B, et al. Variation in clinical phenotype of human infection among genetic groups of Blastomyces dermatitidis. J Infect Dis. 2013;207:814-22.

7. Brown EM, McTaggart LR, Zhang SX, Low DE, Stevens DA, et al. Phylogenetic analysis reveals a cryptic species Blastomyces gilchristii, sp. nov. Within the human pathogenic fungus blastomyces dermatitidis. PLoS ONE. 2013;8:e59237.

8. Imai DM, McGreevey N, Anderson JL, Darien B, Meece JK. Disseminated Blastomyces dermatitidis, genetic group 2, infection in an alpaca (Vicugna pacos). J Vet Diagn Invest. 2014;26:442-7.

9. Bentley DR. The human genome project-an overview. Med Res Rev. 2000:20:189-96

10. Castanón-Olivares LR, Guerena-Elizalde D, Gozalez-Martinez MR, Licea-Navarro AF, Gonzalez-Gonzalez GM, et al. Molecular identification of Coccidioides isolates from Mexican patients. Ann N Y Acad Sci. 2007:1111:326-35.

11. Forche A, Magee PT, Magee BB, May G. Genome-wide single-nucleotide polymorphism map for Candida albicans. Eukaryot Cell. 2004;3:705-14.

12. Syrmis MW, Kidd TJ, Moser RJ, Ramsay KA, Gibson KM, et al. A comparison of two informative SNP-based strategies for typing Pseudomonas aeruginosa isolates from patients with cystic fibrosis. BMC Infect Dis. 2014;14:307.

13. Syrmis MW, Moser RJ, Whiley DM, Vaska V, Coombs GW, et al. Comparison of a multiplexed MassARRAY system with real-time allele-specific PCR technology for genotyping of methicillin-resistant Staphylococcus aureus. Clin Microbiol Infect. 2011:17:1804-10.

14. Muñoz JF, Gauthier GM, Desjardins CA, Gallo JE, Holder J, et al. The dynamic genome and transcriptome of the human fungal pathogen Blastomyces and close relative emmonsia. PLoS Genet. 2015;11:e1005493.

15. Anderson CA. Data quality control. In: Zeggini E, Morris A, editors. Analysis of complex disease association studies: a practical guide. London: Academic; 2011. p. 96-107.

16. Peakall R, Smouse PE. GENALEX 6: genetic analysis in Excel. Population genetic software for teaching and research. Mol Ecol Notes. 2006:6:288-95.

17. Pritchard JK, Stephens M, Donnelly P. Inference of population structure using multilocus genotype data. Genetics. 2000;155:945-59.

18. Evanno G, Regnaut S, Goudet J. Detecting the number of clusters of individuals using the software STRUCTURE: a simulation study. Mol Ecol. 2005:14:2611-20.

19. Earl DA, vonHoldt BM. STRUCTURE HARVESTER: a website and program for visualizing STRUCTURE output and implementing the Evanno method. Conserv Genet Resour. 2012;2:359-61.

20. Butler JM, Coble MD, Vallone PM. STRs vs SNPs: thoughts on the future of forensic DNA testing. Forensic Sci Med Path. 2007;3:200-5.

21. Gill P. An assessment of the utility of single nucleotide polymorphisms (SNPs) for forensic purposes. Int J Legal Med. 2001;114:204-10.

22. McDonough ES, Chan DM, McNamara WJ. Dual infection by "+" and "-" mating types of Ajellomyces (Blastomyces) dermatitidis. Am J Epidemiol. 1977;106:67-71.

\section{Submit your next manuscript to BioMed Central and we will help you at every step:}

- We accept pre-submission inquiries

- Our selector tool helps you to find the most relevant journal

- We provide round the clock customer support

- Convenient online submission

- Thorough peer review

- Inclusion in PubMed and all major indexing services

- Maximum visibility for your research

Submit your manuscript at www.biomedcentral.com/submit 